\title{
Learning Disability Monitor
}

\author{
Rekha Sugandhi \\ Department of \\ Computer \\ MITCOE, Pune
}

\author{
Tejaswini Kasture \\ Department of \\ Computer \\ MITCOE, Pune
}

\author{
Yash Gupta \\ Department of \\ Computer \\ MITCOE, Pune
}

\author{
Ashish Varghese \\ Department of \\ Computer \\ MITCOE, Pune
}

\begin{abstract}
Autism refers to a group of brain development disorders, which are characterized by difficulty in communication and repetitive behaviors. Every autistic child is unique and hence each child should be given special care and attention for his or her development. In today's world it is quite tedious and frustrating for teachers as well as parents to teach autistic children because they have to be taught repeatedly again and again. Due to this many parents actually accept the fact that their child cannot be taught or provided knowledge. Recent development in mobile technologies have provided teachers a better way to teach autistic children through a user friendly GUI and customizable systems deployed on mobile phones. Our proposed system eliminates the repetitive efforts required by teachers and parents by providing a customizable, adaptable and cost efficient system which adapts itself according to the behavior and learning of the child.
\end{abstract}

\section{General Terms}

Autistic, algorithm, learning

\section{Keywords}

Intelligent tutors, $\mathrm{HCI}$, autism

\section{INTRODUCTION}

Autistic children face difficulty in verbal and non-verbal communication that affects the relationship with the world around them. They suffer from complex brain development disorders due to which they tend to forget the things taught to them and find it difficult to grasp anything at one glance; hence they need to be taught repeatedly. Teachers and parents of autistic children play a vital role in teaching them. But practically it is not possible for humans to enrich the child continuously and hence to overcome this situation a system has been proposed which is build using open source tools. This system provides elementary knowledge and does an analysis of the level of learning by considering factors like response time, energy level and the different gestures of the child. Depending on the learning of the child, the system provides the next level of learning. If the child has not gained sufficient knowledge the system adapts according to the learning of the child and provides appropriate knowledge thus eliminating the repeated efforts required by teachers and parents to teach autistic children.

\section{RELATED WORK}

Related work is discussed using the different Human Computer Interaction (HCI), learning levels and approaches used in the previous models

\subsection{Intelligent Tutoring System (ITS)}

B.H Sreenivasa Sarma et al. [26] introduced an intelligent tutoring system which was deployed on desktops to provide basic pattern matching knowledge to autistic children. Here basically a pattern matching question is given to autistic children and they interact with the system using the mouse \& keyboard. These systems are multimodal systems which interact with the child in the form of audio and video and have two levels of learning, Initial learning includes basic pattern matching learning in which a pattern is shown and options are provided from which the child has to select the correct pattern, in the second level of learning many patterns have to be combined together to form a meaningful pattern. ITS applies Reinforcement Learning (RL) algorithm. Fig. 1 shows the testing phase of student using ITS. The RL agent has a function approximator and a RL algorithm. The state of the student is the previous questions asked and the response of the student for those questions. Initially a random state of the student is considered and the reward for the agent is obtained. Depending on the reward and state the agent takes action. The $\mathrm{RL}$ agent has to select the most appropriate question or hint. A reward for the RL is calculated from the student's response. Then the student is trained with same question and state of the student is obtained from the trained output. This process is continued for a number of questions. The main function of the $\mathrm{RL}$ algorithm is that it acts as a pedagogical module which selects appropriate action to teach autistic children. It is concerned with how RL agent should take actions in order to increase its reward. 


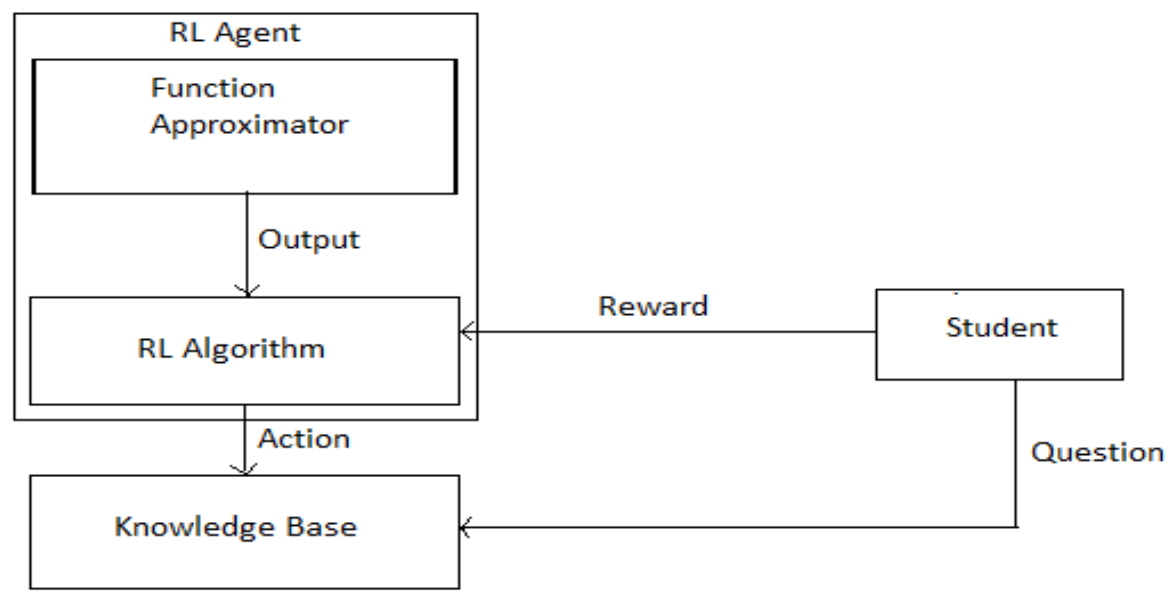

Figure 1 Testing Phase of Student

In case of ITS which uses RL, the algorithm must have (or learn) model of environment, it needs to know where actions lead in order to evaluate actions and make decision. Also the system can be used as a teaching tutor only inside classrooms or houses; it cannot be carried along with us wherever required. Hence it has a drawbacks that it is only restricted to desktops and RL algorithm needs to know the environment model in advance.

\subsection{M-Learning Applications}

M-Learning or mobile learning is a form of distance education where m-learners use mobile device educational technology at their time convenience. It is form of learning across multiple contexts through social and content interaction. Here application is deployed on android devices which are used for increasing the communication skills of the child.

\subsubsection{Prologue 2}

Marry Ann Romski et al. [9] developed Prologue 2 which is an English, French, Spanish symbol supported computer application to promote language development and increase communication skills. It is deployed on I-pads and I-phones in which the autistic child responds in the form of text and this is converted into audio and provided to the autistic child. These particular applications are unimodal systems which provide success in assisting people with communication difficulties. It serves as an AAC (Augmented and alternative communication system) in the pocket and it is quite popular due to its flexible multimedia content storage, portability, mobility and affordability. Prologue 2 is a cost efficient system for imparting communication skills among autistic children but there is no analysis of the level of learning of the child .Provision of knowledge is not just important but an analysis of the learning must be done to provide the most appropriate learning. Hence Prologue 2 has a drawback that it does not do any analysis of the level of learning of the child.

\subsubsection{Picture Exchange Communication System (PECS) on Mobiles}

A. Lema et al. [25] introduced Picture Exchange communication system on smart phones. Picture Cards are used in a training protocol called Picture Exchange
Communication System (PECS). They are multimodal systems which have been the most important functional communication among non-verbal autistic children. These picture cards are specifically made for each individual. In PECS autistic children learn to combine many pictures to convey a message. Traditional tech PECS is quite timeconsuming and inefficient. Researchers have developed the software to emulate the PECS system in which the child is allowed to move the images around to form simple meaningful sentence. Similarly in PECS there is no analysis of the learning of the child .Analysis of the level of learning plays a very vital role after provision of knowledge.

\subsubsection{My Socious}

My Socious is an application deployed on I-pads, I-phones and I-pods which is used for improving the communication skills of autistic children. It uses methods that are natural for teaching the child during its day to day activities .basically it is used for improving the social skills of autistic children. Parents and teachers can see their progress within a month or a year which helps them to make appropriate changes which will be beneficial for autistic children. But the major drawback here is that the application is not cost efficient so it does not benefit the weaker sections of the society which suffer from autism [27].

\subsection{Learning Approaches}

\subsubsection{Decision Trees}

M.S. Mythili et al. [10] made use of decision trees to utilize the learning capability of the machine according to the student. A decision tree is basically a flow chart resembling a tree structure, where each internal node is notated by rectangles. Decision tree is easier to implement and grasp. It starts with a root node that allows the users to take needed actions. From this node, users split each node recursively according to decision tree learning algorithm. The final ultimate result is a decision tree in which every branch is associated with a result.

\section{Advantages:}

- Very simple

- Produces accurate results. 
- Supports incremental learning

- Takes less memory

Disadvantages:

- Longer training time

- Complex representation

- Problem of over fitting

\subsubsection{Support Vector Machine}

Support vector machine (SVM) is a prominent example for pattern classification method with numerous various applications. Support vector machine (SVM) is a new technique for data classification was initially recommended by Vapnik in 1995. SVM analyses data and acknowledge patterns. Primarily used for classification and multivariate analysis. Disadvantages of SVM lie in speed and size, both in training and testing. SVM is a binary classifier. To do a multiclass classification, pair-wise classifications has to be done and they are also computationally expensive

\subsubsection{Naive Bayes}

Nittaya Muangnak et al. [28] use Naive Bayes approach to classify data from the student with learning disabilities. The property consists of age, gender; class level etc.It is a simple Statistical Bayesian Classifier. The Naive Bayes classifier in this context gives an accuracy of 94.23 percent. But this is only for a specific data set. Naive Bayes is not suitable for testing data, because they have to share the data with an attribute which has a numeric value.

\section{Advantages}

- Requires short computational time for training.

- Improves the classification performance by removing the irrelevant features

Disadvantages:

- The Naive Bayes classifier requires a very large number of records to obtain good results.

- Less accurate as compared to other classifiers on some datasets.
Table 1. Comparative Analysis

\begin{tabular}{|c|c|c|c|c|}
\hline $\begin{array}{l}\text { Name of } \\
\text { system }\end{array}$ & $\begin{array}{l}\text { Algorith } \\
\mathrm{m}\end{array}$ & Devices & Input & $\begin{array}{l}\text { Observati } \\
\text { on }\end{array}$ \\
\hline $\begin{array}{l}\text { My } \\
\text { Socious }\end{array}$ & $\begin{array}{l}\text { No } \\
\text { Learning }\end{array}$ & $\begin{array}{l}\text { Tablets } \\
\text { Smart } \\
\text { Phones }\end{array}$ & Touch & Costly \\
\hline ITS & $\begin{array}{l}\text { Reinforc } \\
\text { ement } \\
\text { Learning }\end{array}$ & Desktop & $\begin{array}{l}\text { Using } \\
\text { mouse } \\
\text { and } \\
\text { keyboar } \\
\text { d }\end{array}$ & $\begin{array}{l}\text { Costly and } \\
\text { cannot be } \\
\text { used at all } \\
\text { places. }\end{array}$ \\
\hline PECS & $\begin{array}{l}\text { No } \\
\text { learning }\end{array}$ & $\begin{array}{l}\text { Tablets } \\
\text { Smart } \\
\text { Phones }\end{array}$ & Touch & $\begin{array}{l}\text { No } \\
\text { analysis of } \\
\text { the level } \\
\text { of } \\
\text { learning. }\end{array}$ \\
\hline $\begin{array}{l}\text { Prologue } \\
2\end{array}$ & $\begin{array}{l}\text { No } \\
\text { Learning }\end{array}$ & $\begin{array}{l}\text { Tablets } \\
\text { Smart } \\
\text { Phones }\end{array}$ & Touch & $\begin{array}{l}\text { No } \\
\text { analysis of } \\
\text { the level } \\
\text { of } \\
\text { learning. }\end{array}$ \\
\hline
\end{tabular}

Table 1 shows the Comparative Analysis of the previous systems .The previous systems discussed above which were designed for autistic children were providing knowledge to the autistic children but there was no analysis about how designed for autistic children were providing knowledge to the autistic children but there was no analysis about how much the child has learnt. There is a no use in developing systems which provide knowledge but do not analyze the learning of the child .Hence a system has been proposed built using open source tools which is customizable, adaptable an cost effective, thus overcoming all the previous drawbacks. This system will be deployed on android platform. 


\section{PROPOSED SYSTEM}

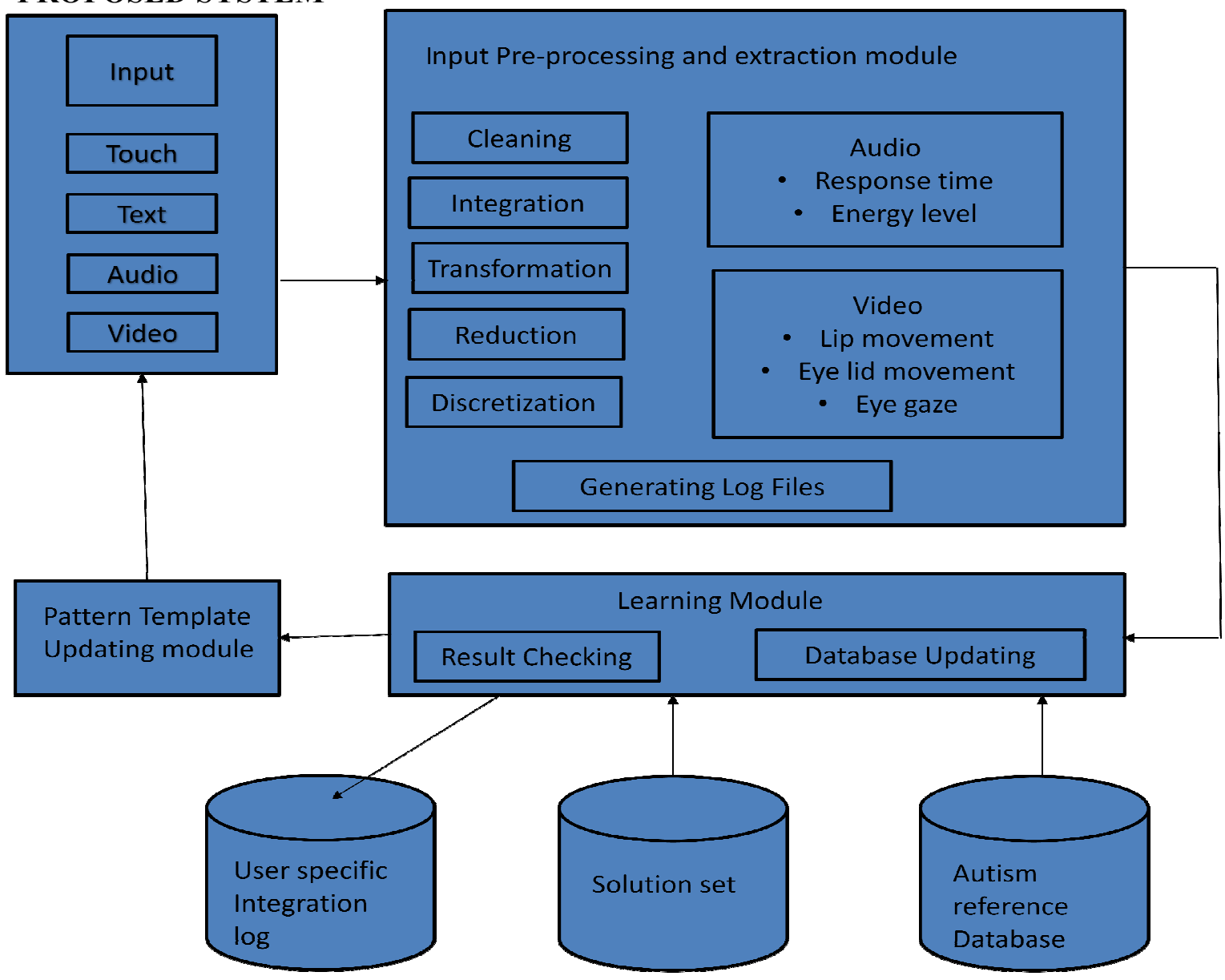

Figure2. Proposed Architecture

\subsection{Data Pre-processing Module}

Real world data such as audio or video from the child contains noise and outliers which makes the data inconsistent, this data cannot be further used hence there is a need of a data preprocessing module which converts the data to consistent and useful data. Data preprocessing contains the following steps

\section{Data Cleaning \\ 2. Data Integration \\ 3. Data Transformation \\ 4. Data Reduction \\ 5. Data Discretization}

\subsubsection{Data Cleaning}

Data cleansing, data cleaning, or data scrubbing is the process of detecting and correcting (or removing) corrupt or inaccurate records from database. The main function is to remove the inconsistent and dirty data.

\subsubsection{Data Integration}

Data integration involves combining data residing on different sources and providing users with a unified view of this data. This process becomes significant in a variety of situations, which include both commercial (when two similar companies need to merge their databases) and scientific (combining research results from different bio informatics repositories)

\subsubsection{Data Transformation}

Data transformation is the process of converting data or information from one format to another, usually from the format of a source system into the required format of a new destination system. Data mapping maps data elements from the source data system to the destination data system and captures any transformation that must occur.

\subsubsection{Data Reduction}

Data reduction is the transformation of numerical or alphabetical digital information into corrected and simplified form. the basic idea is to convert information into meaningful sentences.

\subsubsection{Data Discretization}

It is a part of data reduction which replaces numeric data with nominal ones. This process is usually carried out as first step toward making nominal data suitable for numeric evaluation and implementation on digital computers.

\subsubsection{Generation of Log files}

After preprocessing the data, the data is stored as log files. These log files are tagged for greater efficiency. These log 
files are further used by the learning module for answer comparison.

\subsection{Learning Module}

It compares the input with the solution set. According to the result of the comparison this module decides the difficulty level of the next questions to be asked. The input from the child can be in the form of audio/video/touch/text. The input data will then be pre-processed in which several stages like cleaning, integration, transformation, reduction and discretization. Various gestures of the child will also be considered while he/she is giving the input. Gestures can be in the form of lip movement, eye lid movement and eye gaze through continuous video streaming. For audio, the energy level and response time can be taken into consideration. It also creates and maintains log files which are tagged for better efficiency. Log files are used to store the response of the child to a particular question. The data in the log files is compared with the data in the solution set which is a part of the Learning module in which a template matching is used to determine whether or not the input given by the child is correct and accordingly database is updated. This is a semi-supervised learning approach. The Learning module refers the 'User specific integration log', 'solution set' and 'autism reference database' to adapt to the situation accordingly.

\subsection{Pattern-Template Updation Module}

This module decides by how much level the learning of the child should be increased or decreased. And it frames the next question to be asked.

\subsection{Proposed Algorithm}

The pattern template updation module uses Random Forest algorithm for classification and to decide which question should be given to the child. It also decides what should be the difficulty of that question. Random forest or random decision forest are an ensemble learning method for classification, regression and other tasks, that operate by constructing multiple of decision trees at training time and outputting the classes.

Advantages over other algorithms

- More efficient and accurate.

- Runs efficiently on large data bases.

- It can handle thousands of input variables.

- It gives estimates of what variables are important in the classification.

- Has an efficient method to find out the missing values.

- It takes less training time and has easy representation.

- Generated forests can be saved for future use on other data.

\section{FLOW OF THE SYSTEM}

Teachers and parents cater to the needs of special children and also aid the child to increase their communication skills. The main goal of this system is to provide elementary knowledge and do any analysis of the level of learning of the child. The system accepts input in the form of audio, video, text, image and this input is pre-processed by the data pre-processing module. This module also extracts energy level and response time from the audio using open source tools like ffmpeg which is a command-line tool that converts audio or video formats. Also praat is used for the analysis of speech in phonetics and spectrograms. The system also records the gestures of the child, which further helps in understanding the concentration level of the child. The extracted data is stored in $\log$ files. The learning module compares the input with the solution and also considers factors such as energy level, Pitch and the gestures of the child. Depending on all such factors a rating is given to the answer. For each interaction the user integrated $\log$ is updated. The Pattern Template Matching module uses the Random Forest Algorithm which is used for classification and to decide by how much level the difficulty of the next question should be increased. In such a way the system adapts itself according to the behaviour of the child.

\section{CONCLUSION}

The previous related work shows that the available systems in the market are expensive, unreliable and because of one side communication the learning approaches are not efficient .Hence to overcome such drawbacks Learning disability monitor system has been proposed which is more reliable, cost efficient and adapts according to the learning of the child. Mainly there is analysis of the learning of the child which makes it a very efficient system as compared to the previous ones. From analysis on comparison among data mining classification algorithms it shows that Random Forests algorithm is more accurate. Random Forests does not overfit and is the most efficient for large data sets. On the other hand SVM and decision trees require a lot of training time and they produce accurate results only for specific datasets. The system can be used in the future for providing higher levels of learning.

\section{ACKNOWLEDGMENTS}

We would like to thank BCUD SPPU for giving us this opportunity and funding our project. We would also like to thank MIT College of Engineering and Prasanna Autistic Centre for helping us and guiding us whenever required.

\section{REFERENCES}

[1] N.H. Sweilam, A. Tharwat, A. Abdel, N. K. Moniem, "Support vector machine for diagnosis cancer disease", Egyptian Informatics Journal, Volume (11), pp. 81-92, 2010.

[2] F. Heppner, U. Grenander, "A stochastic nonlinear model for coordinated bird flocks", AAAS publications, Washington, 1990

[3] Tu, Chung-Jui, Li-Yeh Chuang, Jun-Yang Chang, and Cheng-Hong Yang. "Feature selection using PSO-SVM." IAENG International journal of computer science 33, no. 1 (2007): 111-116.

[4] B.T. Chen, M.Y. Chen, "Applying particles swarm optimization for support vector machines on predicting company financial crisis", International Conference on Business and Economics Research, 2010.

[5] N. Arvanitopoulos, D. Bouzas, A. Tefas, "Subclass Error Correcting Output Codes Using Fisher's Linear Discriminant Ratio, Pattern Recognition”, pp. 29532956, 2010.

[6] E.Alba, J. Garcia-Nieto, L. Jourdan, E. Talbi, "Gene selection in Cancer Classification using PSO/SVM and GA/SVM Hybrid Algorithms", Congress on Evolutionary Computation, 2007 
[7] Smruti Rekha Das, Pradeepta Kumar Panigrahi, Kaberi Das,Debahuti Mishra, "Improving RBF Kernel Function of Support Vector Machine using Particle Swarm Optimizaton", International Journal of Advanced Computer Research ,pp 2277-7970,Volume -2,Number 4,Issue-7,December-2012.

[8] M.S. Mythili, A.R.Mohamed Shanavas,"A Study on Autism Spectrum Disorders using classification Techniques",IJSCE,ISSN:2231-2307,Volume 4,Issue 5, Nov 2014.

[9] MaryAnn Romski, PhD,CCC-SLP;Rose A.Sevcik,PhD," Augmentative Communication And Early Intervention", Vol. 18, No. 3, pp. 174-185,2005

[10] M.S. Mythili,, A.R.Mohamed Shanavas," A Novel Approach to Predict the Learning Skills of Autistic Children using SVM and Decision Tree ", Vol. 5 (6), 2014, 7288-729

11) C. E. Pugliese, L. Anthony, J. F. Strang, K. Dudley, G. L. Wallace, and L. Kenworthy, "Increasing Adaptive Behavior Skill Deficits From Childhood to Adolescence in Autism Spectrum Disorder: Role of Executive Function," Journal of Autism and Developmental Disorders, vol. 45, no. 6, pp. 1579-1587, 2015.

12) D. A. Gómez-Aguilar, Á. Hernández-García, F. J. GarcíaPeñalvo, and R. Therón, "Tap into visual analysis of customization of grouping of activities in eLearning," Computers in Human Behavior, vol. 47, pp. 60-67, 2015.

[13] M. H. Charlop-Christy, L. Le, and K. A. Freeman, "A comparison of video modeling with in vivo modeling for teaching children with autism," Journal of Autism and Developmental Disorders, vol. 30, no. 6, pp. 537-552, 2000.

[14] G. R. Hayes, S. Hirano, G. Marcu, M. Monibi, D. H. Nguyen, and M. Yeganyan, "Interactive visual supports for children with autism," Personal and Ubiquitous Computing, vol. 14, no. 7, pp. 663-680, 2010.

[15] M. Monibi and G. R. Hayes, "Mocotos: Mobile communications tools for children with special needs," in Proceedings of the $7^{\text {th }}$ International Conference on Interaction Design and Children, IDC 2008, 2008, pp. $121-124$.

[16] J. P. Hourcade, N. E. Bullock-Rest, and T. E. Hansen, "Multitouch tablet applications and activities to enhance the social skills of children with autism spectrum disorders," Personal and Ubiquitous

[17] H. S. S. Yee, "Mobile technology for children with autism spectrum disorder: Major trends and issues," presented at the 2012 IEEE Symposium on E-Learning, E-Management and E-Services, IS3e 2012, 2012, pp. 6 10.
[18] I. G. Careaga, Evaluación de la eficacia de las intervenciones psicoeducativas en los trastornos del espectro autista. Ministerio de Ciencia e Innovación, Instituto de Salud Carlos sIII, 2009.

[19] E. Husni and Budianingsih, "Mobile Applications BIUTIS: Let's Study Vocabulary Learning as a Media for Children with Autism," Procedia Technology, vol. 11, pp. 1147-1155, 2013

[20] S. Venkatesh, S. Greenhill, D. Phung, B. Adams, and T. Duong, "Pervasive multimedia for autism intervention," Pervasive and Mobile Computing, vol. 8, no. 6, pp. 863882, 2012.

[21] L. DeThorne, B. Aparicio, K. Karahalios, J. Halle, and E. Bogue, "Visualizing Syllables: Real-Time Computerized Feedback Within a Speech-Language Intervention," Journal of Autism and Developmental Disorders, vol. 45, no. 11 , pp. 3756-3763, 2015.

[22] L. Escobedo, D. H. Nguyen, L. A. Boyd, S. H. Hirano, A. Rangel, D García-Rosas, M. Tentori, and G. R. Hayes, "MOSOCO: A mobile assistive tool to support children with autism practicing social skills in real-life situations," presented at the Conference on Human Factors in Computing Systems - Proceedings, 2012, pp. 2589-2598.

[23] S. Bernardini, K. Porayska-Pomsta, and T. J. Smith, "ECHOES: An intelligent serious game for fostering social communication in children with autism," Information Sciences, vol. 264, pp. 41-60, 2014.

[24] J. Mintz, "Additional key factors mediating the use of a mobile technology tool designed to develop social and life skills in children with Autism Spectrum Disorders: Evaluation of the 2nd HANDS prototype," Computers and Education, vol. 63, pp. 17-24, 2013..

[25] A. Lerna, D. Esposito, M. Conson, and A. Massagli, "Long-term effects of PECS on social-communicative skills of children with autism spectrum disorders: a follow-up study," International journal of language \& communication disorders / Royal College of Speech.

[26] B.H Sreenivasa Sarma and B.Ravindran "Intelligent tutoring systems using reinforcement learning to teach autistic children"thesis http://www.cse.iitm.ac.in/ ravi/papers/Sreenivas_thesis.p df

[27] Evelyn Bartlett, Craig Lutz, Roger Bartlett (2012): My Socius

[28] Sayali D.Jadhav and H.P Channe" Comparative Study of K-NN, Naïve Bayes, Decision Tree Classification Techniques "International Journal of Science an d Research 319-7064 